\title{
YAP Promotes Cell Proliferation and Epithelium- Derived Cytokine Expression via NF-kB Pathway in Nasal Polyps
}

\author{
Huiyi Deng (D) ${ }^{1, *}$ \\ Meijiao $\mathrm{Li}^{2}{ }^{2, *}$ \\ Rui Zheng ${ }^{\text {,* }}$ \\ Huijun Qiu' \\ Tian Yuan' \\ Weihao Wang' \\ Qintai Yang' \\ Zijie Long ${ }^{3}$ \\ Xuekun Huang' \\ 'Department of Otorhinolaryngology- \\ Head and Neck Surgery, Department of \\ Allergy, The Third Affiliated Hospital, Sun \\ Yat-sen University, Guangzhou, People's \\ Republic of China; ${ }^{2}$ Department of \\ Otorhinolaryngology-Head and Neck \\ Surgery, Tung wah Hospital of Sun Yat- \\ sen University, Dongguan, People's \\ Republic of China; ${ }^{3}$ Department of \\ Hematology, The Third Affiliated \\ Hospital, Sun Yat-sen University, \\ Guangzhou, People's Republic of China
}

*These authors contributed equally to this work

Correspondence: Xuekun Huang Department of Otolaryngology-Head and Neck Surgery, Department of Allergy, The Third Affiliated Hospital of Sun Yatsen University, Guangzhou, People's Republic of China

Tel +86 020-85252239

Fax +86 020-85253210

Email xuekunhuang@163.com

Zijie Long

Department of Hematology, The Third Affiliated Hospital of Sun Yat-sen

University, Guangzhou, People's Republic of China

Tel $+86020-85253601$

Fax +86 020-85253210

Email longzij@mail.sysu.edu.cn
Background: Hippo-Yes-associated protein (YAP) pathway plays an important role in epithelial cell proliferation and inflammation development in chronic rhinosinusitis with nasal polyps (CRSwNP). However, the underlying mechanisms remain unclear.

Objective: This study intends to investigate the role of YAP and the nuclear factor kappa-B $(\mathrm{NF}-\mathrm{kB})$ pathway in cell proliferation and the expression of epithelium-derived cytokines in nasal polyps (NP).

Methods: The expression levels of YAP, TEA domain family member 1 (TEAD1), Ki-67, and NF- $\kappa \mathrm{B}$ as well as interleukin (IL-) 33, IL-25 and thymic stromal lymphopoietin (TSLP) in sinonasal mucosa, primary nasal epithelial cells (NPECs), and human nasal epithelial RPMI 2650 cells were detected. NPECs were cultured and treated with verteporfin (VP), YAP shRNA or BAY 11-7082.

Results: The hippo pathway effector YAP, Ki-67, p65 NF-kB, and cyclin D1 were significantly increased in NP compared with control mucosa, which was accompanied by overexpression of IL-33, IL-25, and TSLP. Pharmaceutical inhibition of YAP by VP suppressed cell proliferation of RPMI 2650 cells by blocking cell cycle progression at G0/G1 without inducing obvious cell apoptosis. Furthermore, lentiviral transfection-mediated knockdown of hippo pathway activity reduced the expression of IL-33, IL-25, TSLP as

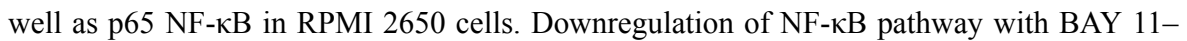
7082 in NPECs could decrease the mRNA level of TSLP, IL-33 and IL-25 accordingly.

Conclusion: Inhibition of hippo pathway suppressed nasal epithelial cell proliferation and declined the expression of epithelium-derived cytokines via the NF- $\kappa$ B pathway in NPECs. Keywords: NF- $\kappa \mathrm{B}$ signalling pathway, yes-associated protein, cell cycle progression, cell proliferation, nasal polyps

\section{Introduction}

Chronic rhinosinusitis (CRS), with an estimated prevalence of $8 \%$ in China, ${ }^{1}$ is defined as chronic inflammation of the nose and the paranasal sinuses. CRS with nasal polyps has been typically characterised by epithelial remodelling, manifested as abnormal epithelial proliferation, basal cell proliferation, a thickened subepithelial basement membrane and fibrosis or squamous metaplasia. ${ }^{2-4}$

The hippo-yes-associated protein (YAP) signalling pathway plays a critical role in the self-renewal and expansion of stem cells and tissue-specific progenitor cells, as well as in tissue regeneration and organ size. ${ }^{5}$ When the hippo pathway lacks of inhibitory phosphorylation, YAP translocates to the nucleus and interacts with 
transcriptional cofactors, including the TEA domain (TEAD) family member of proteins, to turn on proproliferative and pro-survival genes associated with cell proliferation and differentiation. YAP has been implicated in airway epithelial cell proliferation and differentiation. YAP is necessary for regular branching morphogenesis and epithelial cell proliferation as well as differentiation in the developing lung. In addition, transcriptional co-activator with PDZ-binding motif (TAZ), a YAP paralog, was vital to normal alveolarisation in mice. When TAZ is deleted, mice had enlarged abnormal alveoli as human pulmonary emphysema. ${ }^{6}$ Similarly, deletion of MST1/2 in mice resulted in increased nuclear YAP, which led to epithelial hyperplasia and abnormal differentiation of airway epithelial cells. ${ }^{7}$ Recently, we showed that abnormal expression of the hippoYAP pathway contributed to epithelial proliferation and remodelling in CRSwNP. ${ }^{8}$ However, the precise mechanisms of cell proliferation, tissue remodelling, and epithelial inflammation are still unclear.

The nuclear factor-kappa B (NF- $\mathrm{B})$ pathway plays a fundamental role in the regulation and production of proinflammatory cytokines and tissue remodelling in nasal polyps. Chen et al found that interleukin (IL)-17A upregulated the expression of matrix metalloproteinase (MMP)-9 via the NF- $\mathrm{BB}$ pathway in nasal epithelial cells. ${ }^{9}$ Jung et al showed that $\mathrm{p} 65 \mathrm{NF}-\kappa \mathrm{B}$ and $\mathrm{NF}-\kappa \mathrm{B}$-related inflammatory cytokines were expressed in both eosinophilic and noneosinophilic nasal polyps. This indicated that NF- $\mathrm{B}$ had a pivotal role in the pathogenesis of CRSwNP. ${ }^{10} \mathrm{NF}-\kappa \mathrm{B}$ pathway was upregulated in CRSwNP and is related to IL6 and IL- 8 cytokine expression. ${ }^{11}$ In addition, a therapeutic strategy that targeted NF- $\kappa \mathrm{B}$ in Asian patients with nasal polyps could be established. ${ }^{12}$

In this study, we found that abnormal expression of YAP activity related to epithelial proliferation in nasal polyp tissue during the pathogenesis of CRSwNP. In addition, $\mathrm{NF}-\kappa \mathrm{B}$ was increased in CRSwNP tissue and promoted epithelium-derived thymic stromal lymphopoietin (TSLP), IL-25, and IL-33 expression in patients with CRSwNP. Inhibition of YAP-NF- $\mathrm{B}$ could be a therapeutic strategy for reducing epithelial proliferation and epithelium-derived cytokine production in CRSwNP.

\section{Materials and Methods}

\section{Study Subjects and Sample Collection}

This study was conducted in accordance with the Declaration of Helsinki. The consent procedure was approved by the Ethics Committee for Human Study at The Third Affiliated Hospital of Sun Yat-sen University (China). All patients were informed of the purposes and procedures of the study and provided written informed consent. Biopsies of patients with nasal polyps $(n=10)$ and controls $(n=10)$ were recruited from the Department of Otolaryngology in The Third Affiliated Hospital of Sun Yatsen University in China. The demographic information of the study patients is shown in Table 1. The diagnoses and classification of CRS were based on the European Academy of Allergy and Clinical Immunology (EAACI) guidelines (EPOS2012). ${ }^{13}$ All NP patients were bilateral with Grade-3 NP which had completely obstructed the nasal cavity. None of the NP patients and controls had a concurrent upper respiratory infection, asthma and other systemic diseases. In addition, any form of glucocorticosteroids or antibiotics within three months were did not be used before the study. Biopsies were obtained during functional endoscopic sinus surgery (FESS). Biopsies of inferior turbinate (IT) mucosa were obtained from the control patients, who had no history of CRS and no evidence of sinonasal mucosal inflammation on endoscopy but were undergoing sinonasal surgery for other indications, such as deviation of septum, pituitary tumors, encephaloceles, or repair of cerebrospinal fluid rhinorrhea.

Parts of the fresh nasal tissues were used for quantitative reverse transcription-polymerase chain reaction (qRTPCR) and histological analysis, and some of the tissues were isolated for cell culture.

\section{Immunohistochemistry in Solid Tissues}

Tissue samples were fixed in 10\% neutral buffered formalin and embedded in paraffin. Paraffin sections $(4 \mu \mathrm{m})$ were prepared for each block. Sections were subjected to heat-induced antigen retrieval. Endogenous peroxidase activity was blocked with $0.3 \%$ hydrogen peroxide. Nonspecific staining was blocked with $5 \%$ bovine serum albumin (Boster Biotechnology). Sections were conducted

Table I Patient Characteristics and Histopathological Evaluation

\begin{tabular}{|l|l|l|}
\hline & Controls & CRSwNP \\
\hline Sample number & 10 & 10 \\
Average Age (Range) & $28(22-47)$ & $40(19-50)$ \\
Gender (Male/Female) & $5 / 5$ & $6 / 4$ \\
Smoking history & 2 & 4 \\
Asthma & 0 & 0 \\
Allergic rhinitis & 0 & 0 \\
\hline
\end{tabular}


with specific primary antibodies YAP (1:100, CST, \#14074), NF-kB (1:100, Servicebio, GB11142) and AntiCyclin D1 antibody $(1: 100$, Abcam, ab16663) as previously reported. ${ }^{8}$

\section{Immunoblotting}

Total protein from the treated cells and tissues were extracted by RIPA buffer with the mixture consisting of protease Inhibitor Cocktail (Beyotime, Shanghai, China). Protein was separated by an SDS-PAGE gel and transferred to polyvinylidene difluoride membranes (Millipore, IPVH10100). After blocking with 5\% bovine serum albumin (BSA) for 75 minutes, the PVDF membrane was incubated with primary antibodies (YAP Rabbit mAb (1:1000, CST, \#14074), TEAD1 Rabbit mAb (1:1000, CST, \#12292), Anti-Ki67 Rabbit pAb (1:1000, Servicebio, GB11030), NF-кB p65 (D14E12) $\mathrm{XP}^{\circledR}$ Rabbit mAb (1:1000, CST, \#8242), Anti-Cyclin D1 antibody (1:1000, Abcam, ab16663), Anti-CDK4 antibody (1:1000, Abcam, ab108357, Phospho-IкB $\alpha$ (Ser32/ 36) (5A5) Mouse mAb (1:1000, CST, \#9246), IкB $\alpha$ (112B2) Mouse mAb (Carboxy-terminal Antigen, 1:1000, CST, \#9247) and rabbit monoclonal antiGAPDH antibody (1:3000, Bioworld, AP0063) at $4{ }^{\circ} \mathrm{C}$ overnight. After washing for three times, the membrane was incubated with HRP-conjugated secondary antibodies for $1 \mathrm{~h}$ at room temperature. Finally, enhanced chemiluminescence (Advansta, K-12045-D50) was used to visualize the immunoblot. The intensity of the bands was determined by Image $J$ software. The ratio of each band/GAPDH was considered as the expression level of the target protein.

\section{RNA Extraction and Real-Time Polymerase Chain Reaction Analysis}

The quantitative real-time PCR was performed as previously reported. ${ }^{8}$ All primers used are listed in Table 2. The total RNA $(1 \mu \mathrm{g})$ of each tissue sample was extracted using RNAiso Plus (TaKaRa, Japan) and reversetranscribed to cDNA with random hexamer primers and RT-PCR kits (TaKaRa RR047A, Japan). The YAP, IL-25, IL-33 and TSLP were detected. PCR was performed with an ABI 7500 FAST instrument (Foster City, CA, USA) using an SYBR Premix Ex Taq kit (TaKaRa DDR420A, Japan). The relative fold increase of gene expression was calculated using the comparative $2-\Delta \Delta \mathrm{Ct}$ method. $\beta 2$
Table 2 Primer Sequences for Real Time Quantitative PCR

\begin{tabular}{|l|c|}
\hline Gene Name & Primer Sequence $\left(\mathbf{5}^{\prime}\right.$ '- $\mathbf{3}^{\prime}$ ) \\
\hline$\beta_{2} \mathrm{M}$ & $\begin{array}{c}\text { TACACTGAATTCACCCCCAC } \\
\text { CATCCAATCCAAATGCGGCA }\end{array}$ \\
\hline TSLP & $\begin{array}{c}\text { CCCAGGCTATTCGGAAACTCA } \\
\text { ACGCCACAATCCTTGTAATTGTG }\end{array}$ \\
\hline IL-33 & $\begin{array}{c}\text { AGCCTAGATGAGACACCGAATT } \\
\text { GGTCAGAAGGGATGGTAGGC }\end{array}$ \\
\hline IL-25 & CCAGGTGGTTGCATTCTTGG \\
& TGGCTGTAGGTGTGGGTTCC \\
\hline
\end{tabular}

microglobulin $(\beta 2 \mathrm{M})$ was used as the housekeeping gene for normalization.

\section{Human Nasal Epithelial Cells and RPMI 2650 Culture}

Human nasal epithelial cell culture was performed as previously reported. ${ }^{8}$ Human nasal epithelial cell from fresh specimens had ethical review board approval. Fresh specimens from controls and nasal polyp tissues were immediately washed with cold phosphate-buffered saline supplemented by penicillin, streptomycin and amphotericin repeatedly, which were then cut into pieces and digested by Dispase II (Sigma, St. Louis, MO, D4693) dissolved with DMEM/F-12 (Hyclone, SH30023.01) at $4^{\circ} \mathrm{C}$ overnight. A single-cell suspension was obtained after digesting with pancreatin at $37^{\circ} \mathrm{C}$ for 15 minutes and grinding with 1640 medium (Thermo Scientific) containing 10\% fetal bovine serum (FBS). Cells were seeded and cultured with Serum- and BPE-free medium (STEMCELL, \#05008) at $37^{\circ} \mathrm{C}$ for about 10 days to $80 \%$ confluence. These cells were then treated with Verteporfin, Selleck, S1786) for 24 h or BAY 11-7082, a NF- $\mathrm{kB}$ small molecule inhibitor, for $24 \mathrm{~h}$. RPMI 2650 (Sigma Aldrich), a nasal epithelial cell line, was cultured in 1640 medium with $10 \% \mathrm{FBS}$ at $37^{\circ} \mathrm{C}$ in an atmosphere of $5 \% \mathrm{CO}_{2}$.

\section{Construction of the Lentiviral Interference Vectors and Transfection}

Sequence of the shRNA targeting YAP was designed as follows: 5'-GCCACCAAGCTAGATAAAGAA-3' as described by Rosenbluh et al. ${ }^{14}$ Non-silencing shRNA (5'TTCTCCGAAC GTGTCACGT-3') was synthesized as the negative control (NC shRNA). The shRNA was then 
ligated into lentiviral interference vector, pHBLV-U6MCS-CMV-ZsGreen-PGK-PURO. When HEK 293T cells were transfected with the lentiviral packaging plasmids (pSPAX2, pMD2G, and YAP shRNA) using the LipoFiter ${ }^{\mathrm{TM}}$ Liposomal Transfection Reagent (Hanbio Biotechnology, Shanghai, China), and the viral particles (YAP shRNA) were collected. Finally, both the lentivirus was extracted and concentrations were verified.

For lentivirus transfection, the RPMI 2650 cells were grown to $50-70 \%$ confluency and infected with YAPshRNA and the control shRNA with polybrene at a concentration of $8 \mu \mathrm{g} / \mathrm{mL}$. Cells were washed and switched into the complete medium after transfection after $8 \mathrm{~h}$. The fluorescence was used to confirm the successful transfection of YAP-shRNA lentiviral vectors $2 \mathrm{~d}$ after transfected. The YAP-shRNA infected cells were detected as GFP-positive cells. Stable cell lines containing YAP-shRNA or NC vector were selected using $2.5 \mu \mathrm{g} / \mathrm{mL}$ puromycin (Beyotime, China).

\section{Immunofluorescence Staining in Cell Cultures and Confocal Microscopic Imaging}

Immunofluorescence staining was performed as previously reported. ${ }^{8}$ RPMI 2650 cells were cultured on coverslips and treated with VP for $24 \mathrm{~h}$ when grown to $80 \%$ confluence. After the VP treatment, cells were washed in PBS buffer twice. Next, the treated cells were fixed by $4 \%$ paraformaldehyde at room temperature for 10 minutes. Subsequently, cells were treated with $0.1 \%$ Triton X-100 for 5 minutes at room temperature to permeabilize cells membranes. After nonspecific blocking, the cells were blocked in TBST and 1\% Bovine Serum Albumin at room temperature for 90 minutes, then the cells were incubated with primary antibody (Anti-NFkBp65 Rabbit pAb (1:200, Servicebio, GB11142) overnight at $4^{\circ} \mathrm{C}$. Cells were next incubated with secondary antibody (1:500) conjugated with Alexa Fluor 488-conjugated goat anti-rabbit IgG (Life Technologies, GrandIsland, NY, BIOTIUM20012) for $2 \mathrm{~h}$ at $37^{\circ} \mathrm{C}$ in dark. Cell nuclei were counterstained with SlowFade Gold antifade reagent with 49,6-diamidino-2-phenylindole (DAPI) (Thermo Scientific, D1306). The slides were then analyzed with a confocal microscope (Nikon C2, Japan).

\section{Colony Formation Assay}

RPMI 2650 cell lines, shYAP or NC stable cells, and NPECs were seeded and cultured into the six-well plate in DMEM containing 10\% FBS or with serum-free bronchial epithelial cell growth medium (BEGM) (Lonza, CC-3171) for 24 $\mathrm{h}$. Then, the cells were treated with various concentrations of VP $(0,10 \mu \mathrm{M}$ for RPMI 2650 cell; $0,2.5 \mu \mathrm{M}$ for NPECs). After the treatment, the cells were cultured in $5 \% \mathrm{CO}_{2}, 37^{\circ} \mathrm{C}$ for 7 days to allow colony formation. The colonies were fixed by $4 \%$ paraformaldehyde at room temperature for 10 minutes. Then dyed with $0.1 \%$ crystal violet (Solarbio, China) at room temperature for 15 minutes. Colonies were photographed using BioRad GS-800 Calibrated Densitometer. The colonies and counted by Image $\mathrm{J}$ software. The experiment was repeated three times.

\section{5(6)-Carboxyfluorescein Diacetate Succinimidyl Ester (CFSE) Labelling and Flow Cytometry Assay}

Primary epithelial cells from nasal polyps (NPECs, $1 \times 10^{6}$ cells $/ \mathrm{mL}$ ) or RPMI 2650 cells were washed twice with PBS and collected. The cells were resuspended in $500 \mu \mathrm{L}$ PBS buffer and stained with $5 \mu \mathrm{M}$ CFSE (Biolegend, USA, 423801) for 10 minutes at $37^{\circ} \mathrm{C}$ in dark. Then cells were treated with 10 volumes of ice-cold culture medium (10\% FBS) and were washed with cold PBS buffer twice to remove excess dyes and seeded in 6-well plates for $24 \mathrm{~h}$. Next, the cells were treated with various concentrations of VP $(0$, $10,15,20 \mu \mathrm{M}$ or $0,2.5,5,10 \mu \mathrm{M})$ for $24 \mathrm{~h}$. After the treatment, the percentage of cell proliferation was analyzed by using flow cytometry (BD, FACSCalibur, USA) to evaluate the CFSE fluorescence intensity. The experiment was done for three times.

\section{Flow Cytometry Assay of Cell Cycle and Apoptosis}

$24 \mathrm{~h}$ after VP treatment at different concentration $(0,10,15$, $20 \mu \mathrm{M})$, RPMI 2650 cells were trypsinized and then centrifuged at $1500 \mathrm{rpm}$ for 5 minutes, followed by wash in cold PBS twice. Next, 70\% cold ethanol was added and cells were fixed for more than $2 \mathrm{~h}$. A total of $250 \mu \mathrm{L} \mathrm{PI/RNase} \mathrm{buffer}$ was added and incubated for 30 minutes at RT. Finally, cells analyzed on a flow cytometer (BD Biosciences).

For apoptosis analysis, cells were stained with the Annexin V-FITC/PI apoptosis detection kit (Bestbio, China). The rate of apoptotic cells was analyzed using a dual laser flow cytometer and estimated using the ModFit software (BD Biosciences). 


\section{Cell Counting Kit-8 (CCK8) Assay}

Cell viability analysis was measured by Cell Counting Kit- 8 (CCK8, Beyotime, Shanghai, China) according to the manufacturer's protocols. NPCEs were collected and cultured at a density of $2 \times 10^{3} /$ well into 96 -well. Next, the cells were treated with different concentrations of (Verteporfin, 0, 1.25, $2.55,10,15,20,30,40 \mu \mathrm{M}$, Selleck, S1786). After treatment for $6,12,24$ and $48 \mathrm{~h}$, respectively, $10 \mu \mathrm{L}$ of CCK-8 reagent was added to every well and then cultured for $2 \mathrm{~h}$ in dark. All experiments were performed for three times. The absorbance analysis was using a microplate reader at $450 \mathrm{~nm}$ (Bio-Rad, Hercules, CA, USA).

\section{Statistical Analysis}

All data are presented as means and standard error (mean \pm SD) from the indicated number of samples. If not normally distributed, data were expressed as median. In continuous variables, for non-normally distributed data, the KruskalWallis H-test was used to assess significant intergroup variability. Normal data were assessed by one-way ANOVA for multiple comparisons. The Mann-Whitney $U$ 2-test was applied for non-normally distributed data or Student's $t$ test was used for unpaired data between two groups analysis.
Statistical analysis was performed using IBM SPSS software (SPSS, Inc, Chicago, IL, USA), and GraphPad Prism 6 (GraphPad Software, San Diego, CA, USA). Two-tailed $P$ value less than 0.05 indicates as statistical significance.

\section{Results}

\section{Aberrant Expression of YAP, Epithelial Cell-Derived Cytokines, and Cyclin DI in CRSwNP}

Firstly, we explored the expression of YAP, the cell proliferation marker Ki-67, and NF- $\mathrm{KB}$ in polyp tissues by Western blotting analysis. We found that the protein levels of YAP, Ki-67, and NF-kB were significantly increased in nasal polyp tissues compared with control nasal tissues (Figure 1A and B). Since cyclin D1 is related to cell proliferation, ${ }^{15}$ we detected the expression of YAP, NF$\kappa \mathrm{B}$, and cyclin D1 by immunohistochemistry in nasal polyps and normal control tissues. We found that the expression levels of YAP, NF- $\kappa \mathrm{B}$, and cyclin D1 were significantly increased in nasal polyps. Consistently, YAP, NF- $\kappa B$, and cyclin D1 were mainly upregulated in the epithelium, and low levels were found in the lamina
A

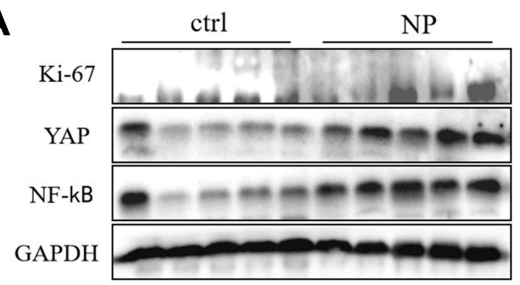

C

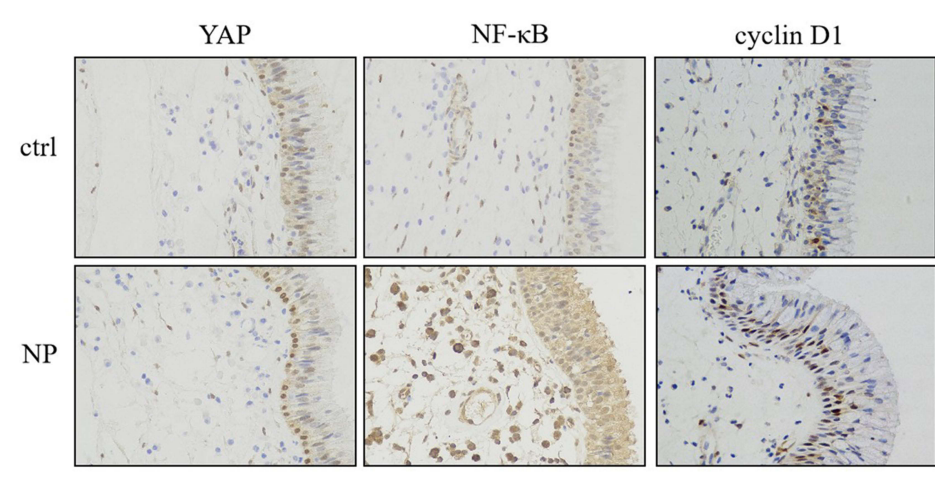

B

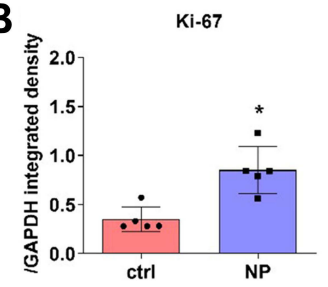

\section{D}
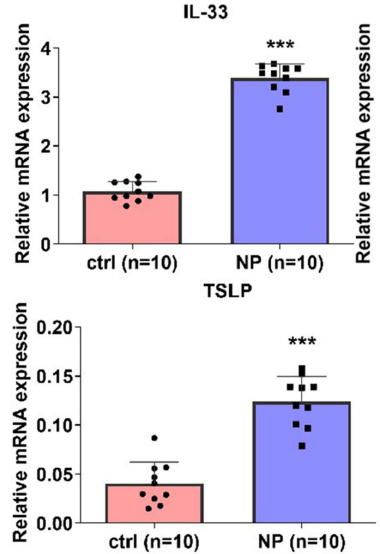

Figure I Expression of hippo pathway effector YAP, NF- $\kappa B$ and epithelium-derived factor in healthy controls and nasal polyps. (A-B) Western blotting analysis for YAP, Ki67, p65 NF-kB and GAPDH expression in total protein extracted from nasal tissues. Representative images from each group are shown. All the bands on the panels were quantified by densitometric analysis using Image J software, and data were expressed as relative values against GAPDH ( $n=5$ for each group, Independent-Sample $t$ test). (C) Immunohistochemical staining for YAP in polyp tissues and healthy control tissues. Representative images from each group are shown ( $\mathrm{n}=10$ for each group, $400 \times$ magnification). (D) Quantitative analysis of transcript levels of epithelium-derived factor including TSLP, IL-33 and IL-25 relative to the expression level of $\beta 2$ microglobulin $(\beta 2 \mathrm{M})$ reference gene in nasal polyp tissues as well as healthy control tissues $(n=10$ for each group, Mann-Whitney $U 2$-test). Data are presented as mean \pm SD. $* P<0.05$, $* * p<0.01$, $* * * P<0.001$. 
propria in nasal polyps (Figure 1C). Moreover, to explore the levels of epithelium-derived innate cytokines, we determined the mRNA levels of TSLP, IL-33, and IL-25 by RT-qPCR. The results showed that the mRNA levels of TSLP, IL-33, and IL-25 were significantly upregulated in nasal polyps compared with those of normal control tissues (Figure 1D). Together, these results indicated that the hippo pathway effector YAP might be involved in epithelium-derived cytokine expression and inflammation via the $\mathrm{NF}-\kappa \mathrm{B}$ pathway in nasal polyps.

\section{Inhibition of YAP Suppresses the Proliferation of RPMI 2650 Cells}

To investigate the impact of YAP on cell proliferation, different density gradients of verteporfin (VP) were used to treat RPMI 2650 cells. We assessed cell viability at 24 $\mathrm{h}$ using a CCK-8 assay. Compared with the control group, a dose-dependent decrease in cell viability was observed in the VP group in (Figure 2A). As shown in Figure 2B, the cell morphology changed after VP treatment. We further examined the proliferation of the three VP-treated cell groups $(10,15$, and $20 \mu \mathrm{M})$ by cell counting. After inhibiting YAP expression in RPMI 2650 cells for 24 and $48 \mathrm{~h}$, we found that there was a significant reduction in cell proliferation (Figure 2C). The results demonstrated that downregulation of YAP led to suppression of epithelial cell proliferation. Further testing using 5(6)carboxyfluorescein diacetate succinimidyl ester (CFSE) labelling was performed to confirm the ability of the proliferation inhibition of VP. The results revealed that the proliferation of cells treated with $15 \mu \mathrm{M}$ and $20 \mu \mathrm{M}$ VP was greatly reduced (Figure 2D and E). Colony formation assays also showed much less colony formation in the group treated with $10 \mu \mathrm{M}$ VP compared with the control group (Figure 2F and G). Moreover, the Western blotting results revealed that VP considerably decreased the expression levels of YAP and TEAD1 in a dosedependent manner, together with Ki-67 (Figure 2H). Thus, pharmaceutical inhibition of YAP suppressed the proliferation and colony formation ability of RPMI 2650 cells.

\section{Inhibition of YAP Arrests the Cell Cycle}

After RPMI 2650 cells were treated with different concentrations of VP, the effect of YAP on cell cycle progression was analysed by FCM In the $20 \mu \mathrm{M}$ VP group, we found that $83.81 \%$ of cells were in G1 phase and
$7.93 \%$ were in S phase. In comparison, $86.12 \%$ of cells were in G1 phase and $8.58 \%$ were in S phase in the 15 $\mu \mathrm{M}$ group, $77.92 \%$ of cells were in G1 phase and $11.67 \%$ of cells were in $\mathrm{S}$ phase in the $10 \mu \mathrm{M} \mathrm{VP}$ group, and $58.87 \%$ of cells were in G1 phase and $31.52 \%$ of cells were in $\mathrm{S}$ phase in the control group (Figure $3 \mathrm{~A}$ and $\mathrm{B}$ ). In addition, we further explored the protein levels of cyclin D1 and cyclin-dependent kinase (CDK) 4, which are involved in cell cycle regulation. Western blot analysis indicated that both cyclin D1 and CDK4 levels were decreased after VP treatment compared with the untreated group (Figure 3C).

Furthermore, the apoptosis rate of cells treated with VP was analysed by FACS. The results showed that in RPMI 2650 cells with pharmaceutical inhibition of hippo pathway activity, the apoptosis rate was slightly increased compared with the group without VP treatment. However, the difference between the control and the $20 \mu \mathrm{M}$ VP group was not significant $(P>0.05$, Figure $3 \mathrm{D}$ and $\mathrm{E})$. These findings suggested that downregulation of YAP induced G1 phase arrest, resulting in suppression of cell proliferation.

\section{Inhibition of YAP Suppresses NF- $\kappa B$ Pathway and Epithelium-Derived Factor Expression in RPMI 2650 Cells}

To further explore whether pharmacological inhibition of hippo pathway activity affects the NF- $\mathrm{KB}$ pathway and epithelium-derived factor expression in epithelial cells, we treated RPMI 2650 cells with VP, and knockdown YAP using lentiviral transfection. Western blotting showed that the expression of NF- $\mathrm{BB}$ decreased in RPMI 2650 cells treated with VP in a dose-dependent manner (Figure 4A and B). Immunofluorescence analysis confirmed the expression of NF- $\mathrm{KB}$ in the epithelial cells of nasal polyps treated with $20 \mu \mathrm{M}$ VP (Figure 4C). Previous study showed that epithelial cells produced epithelium-derived factors, ${ }^{16}$ and we found that VP significantly decreased the mRNA levels of IL-33, TSLP, and IL-25 in a dose-dependent fashion (Figure 4D-F). As expected, both Ki-67 and TEAD1 were significantly decreased after YAP knockdown (Figure 5A). Cell proliferation, colony formation, and IL-33, TSLP, and IL-25 mRNA expression were also decreased (Figure 5B-H). The data demonstrated that inhibition of the hippo pathway by VP or lentiviral transfection could suppress the NF- $\kappa \mathrm{B}$ pathway and epithelium-derived factor expression in RPMI 2650 cells. 
A

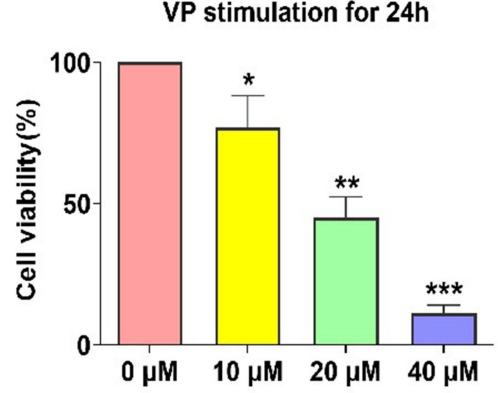

B
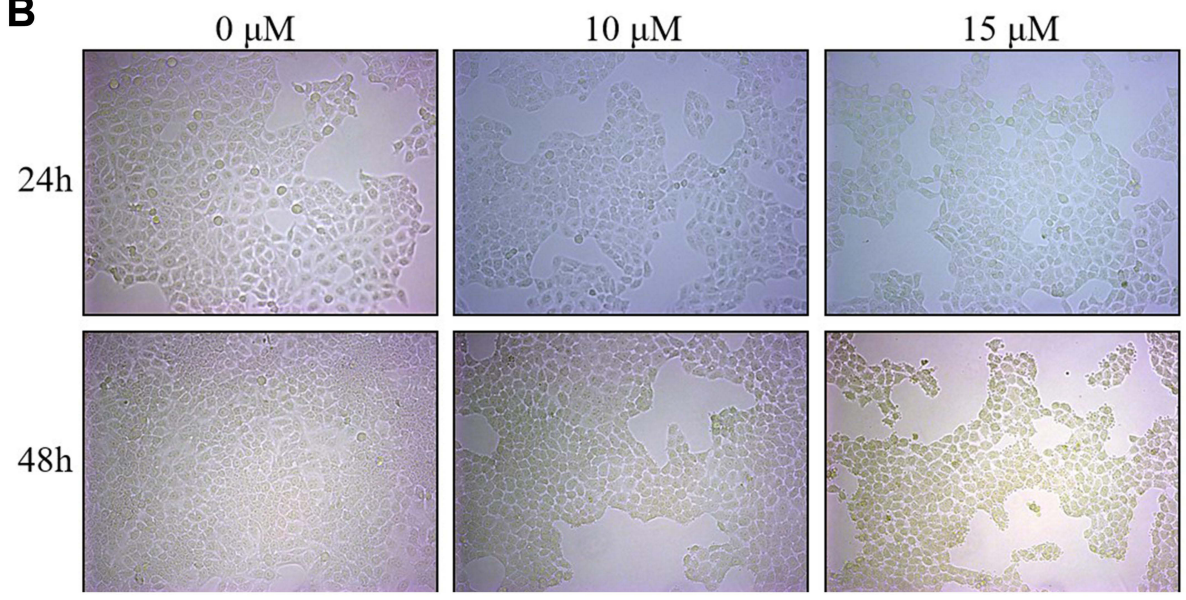

E

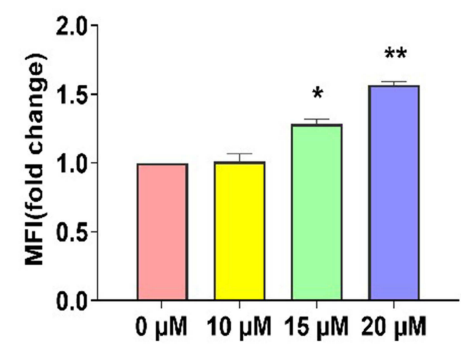

F
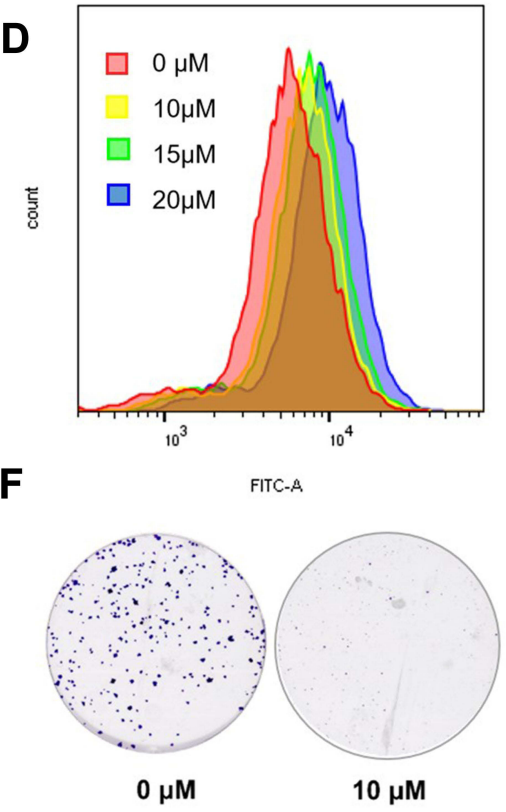

G

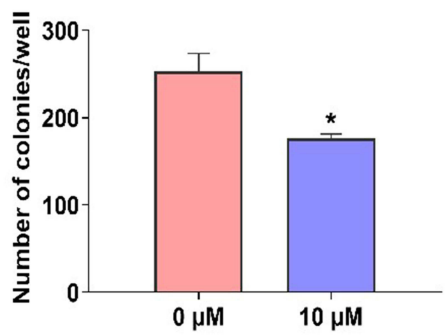

H

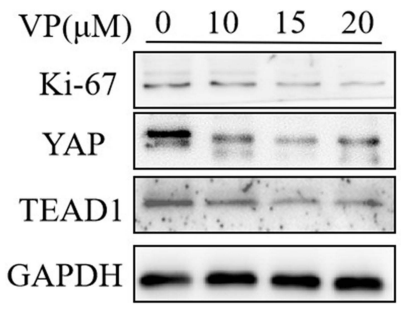

Figure 2 Pharmaceutical inhibition of hippo pathway activity suppresses cell proliferation of RPMI 2650 cells. (A) Cell growth curve showed the cell viability of RPMI 2650 cells treated with verteporfin for $24 \mathrm{~h}$ in different density gradient via CCK-8 assay. (B) RPMI 2650 cells were incubated under verteporfin treatment for 24 and $48 \mathrm{~h}$ in different density gradient. Morphologic change was photographed at $200 \times$ magnification ( $n=3$ for each group). (C) Cell count was measured in cells treated with verteporfin ( $0,10,15,20 \mu M)$ using trypan blue stain assay ( $\mathrm{n}=3$ for each group, one-way ANOVA). (D-E) CFSE cytometric analysis of cell proliferation after VP treatment for $24 \mathrm{~h}$ in various density gradient $(\mathrm{n}=3$ for each group). Quantification of mean fluorescence intensity (MF) in different group ( $n=3$ for each group, one-way ANOVA). (F-G) The ability of proliferation was measured by plate colony-forming assay. Representative images from each group are shown by scanner. The cell counting was measured by counting clone number growing in plate using Image $\mathrm{J}$ software ( $\mathrm{n}=3$ for each group, Independent-Sample $t$ test). (H) Protein levels of YAP, TEADI and Ki-67 were detected in RPMI 2650 cells treated with verteporfin for $24 \mathrm{~h}$ ( $\mathrm{n}=3$ for each group). Results are the mean \pm SD. $* P<0.05$, $* * P<0.01$, $* * * P<0.001$. 

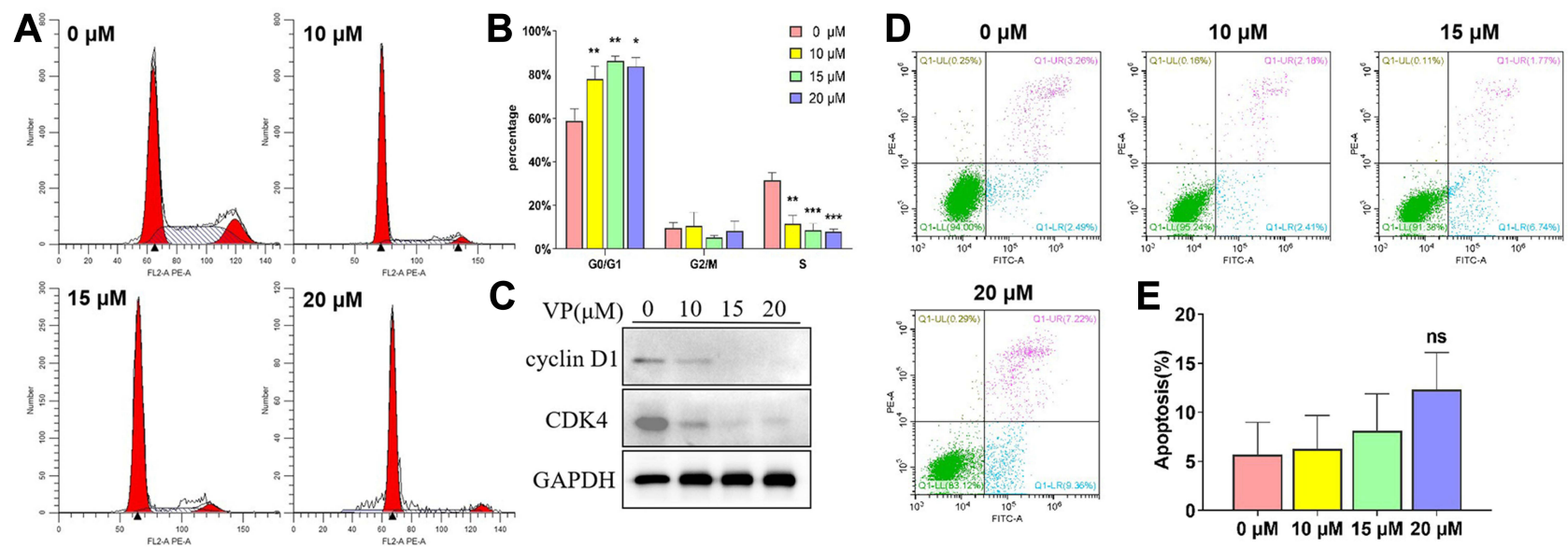

Figure 3 Inhibition of YAP induces cell cycle arrest in RPMI 2650 cells. (A) RPMI 2650 cells treated with VP (0, 10, 15, $20 \mu$ M) after $24 \mathrm{~h}$ and subjected to cell cycle detection. (B) The proportion of cells in each phase was shown ( $\mathrm{n}=3$ for each group, one-way ANOVA). (C) Representative Western blotting analysis of cell cycle progression proteins cyclinDI and CDK4 in RPMI 2650 cells treated with VP for $24 \mathrm{~h}$ ( $\mathrm{n}=3$ for each group). (D) RPMI 2650 cells were treated with VP (0, I0, I5, $20 \mu \mathrm{M}$ ) for $24 \mathrm{~h}$ and the apoptosis was detected ( $\mathrm{n}=3$ for each group). (E) The proportion of apoptotic cells treated with VP of different concentrations after $24 \mathrm{~h}$ was shown ( $\mathrm{n}=3$ for each group, one-way ANOVA). Data were presented as mean \pm SD. $* P<0.05$, $* * p<0.01, * * * P<0.001$.

\section{A}

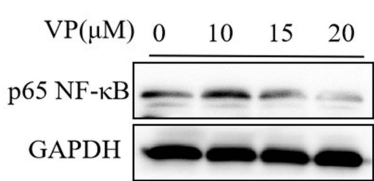

D

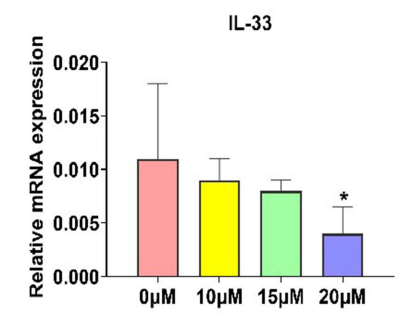

B

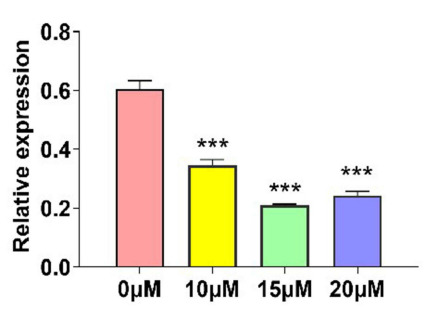

$\mathbf{E}$

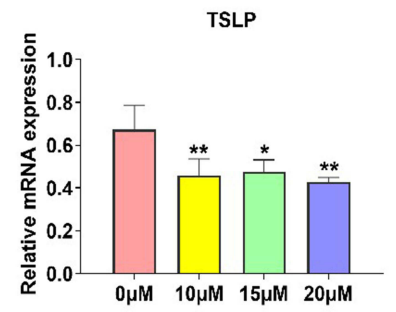

C

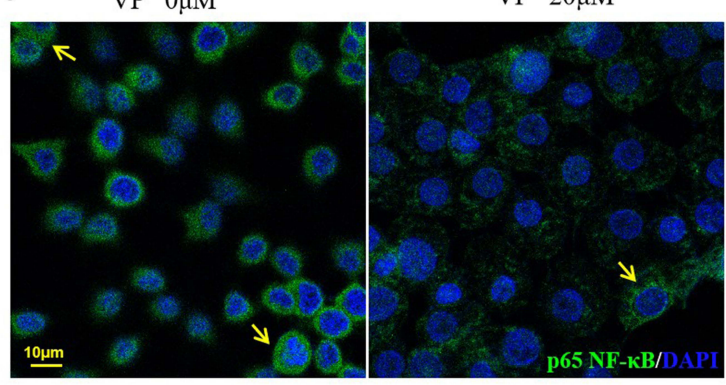

$\mathbf{F}$

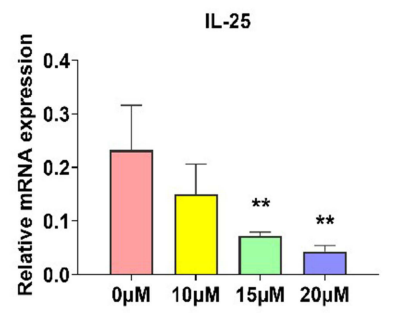

Figure 4 Pharmaceutical inhibition of hippo pathway activity suppresses NF- $\mathrm{B}$ pathway and epithelium-derived factor expression in RPMI 2650 cell. (A-B) Western blotting

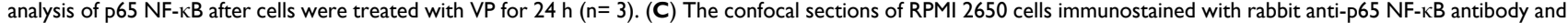
DAPI. Representative confocal images from each group are shown ( $n=3$ for each group, 630xmagnification). (D-F) Quantitative analysis of transcript levels of and epithelium-derived factor including TSLP, IL-33 and IL-25 in RPMI 2650 cells ( $\mathrm{n}=3$ for each group, Kruskal-Wallis H-test). Data are presented as mean \pm SD. $* P<0.05$, $* * P<$ 0.01 , $* * * P<0.001$.

\section{YAP Induces Epithelium-Derived}

\section{Cytokine Expression via NF- $\kappa B$ Signalling in Epithelial Cells Derived from Nasal \\ Polyp Tissues}

We used epithelial cells derived from nasal polyp tissues to confirm our above results. We found that $2.5 \mu \mathrm{M}$ VP effectively hampered hippo-YAP signalling in different periods and different concentrations (Figure 6A). VP also inhibited the expression of NF- $\mathrm{KB}$ and the proliferation of epithelial cells derived from nasal polyp tissues (Figure 6B-G). Moreover, the mRNA levels of TSLP, IL-33, and IL-25 were significantly downregulated with VP treatment compared with those of cells from normal control tissues (Figure 6H-J). Furthermore, BAY 11-7082 was employed 

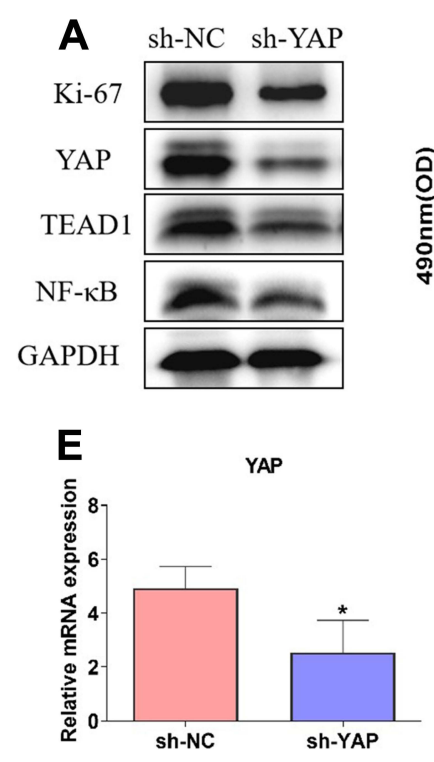
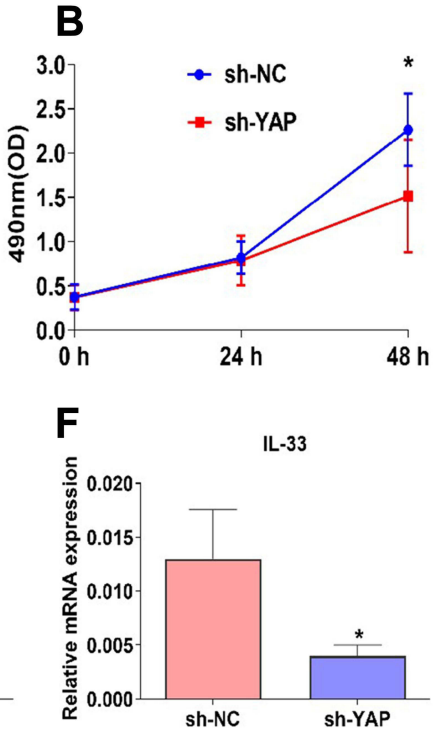

C
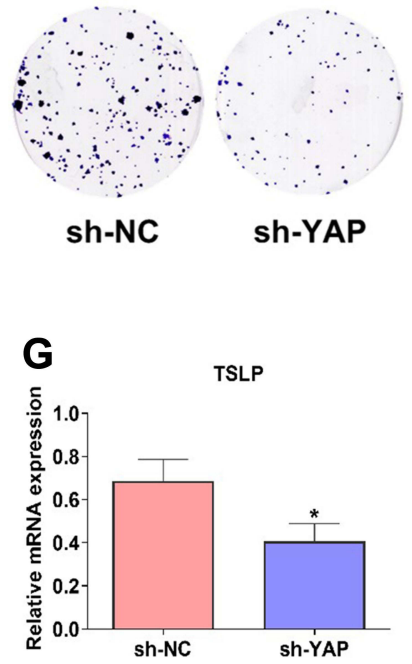
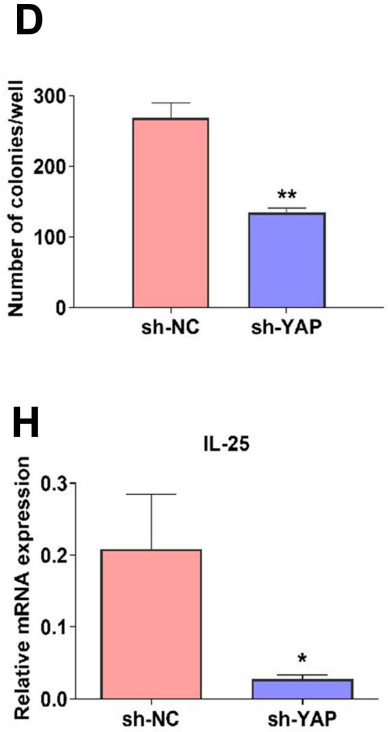

Figure 5 Lentiviral transfection mediated knockdown of YAP results in suppression of cell proliferation properties, and decreased expression of epithelium-derived factors. (A) Protein levels of Ki-67, YAP, TEADI and p65 NF-kB in YAP knockdown cells $(n=3)$. (B) CCK-8 assay was used to evaluate the effect of YAP knockdown on cell proliferation of RPMI 2650 cells after 24 and $48 \mathrm{~h}$ ( $\mathrm{n}=3$ for each group, Independent-Sample $t$ Test). (C-D) The ability of cell proliferation was measured by colony-forming assay. Representative images from each group are shown ( $n=3$ for each group, Independent-Sample $t$ Test). (E-H) YAP, TSLP, IL-33 and IL-25 mRNA levels by RT- PCR in RPMI 2650 cells ( $n=3$ for each group, Mann-Whitney $U 2$-test). Data are presented as mean \pm SD. $* P<0.05, * * P<0.01$.

to reconfirm the expression of VP-induced epithelium-

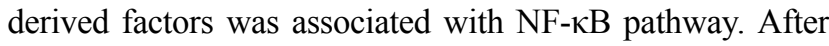
treating NPECs with BAY 11-7082 for $24 \mathrm{~h}$, the protein levels of $\mathrm{pI} \kappa \mathrm{B} \alpha$ and $\mathrm{NF}-\kappa \mathrm{B}$ were attenuated significantly (Figure $6 \mathrm{~K}$ and L). Following treatment with $5 \mu \mathrm{M}$ BAY 11-7082 for $24 \mathrm{~h}$, the mRNA levels of TSLP, IL-33, and IL25 were decreased accordingly (Figure 6M-O), suggesting that the decreased levels of TSLP, IL-33, and IL-25 with inhibition of YAP were mediated by NF- $\kappa$ B suppression.

\section{Discussion}

In our previous study, we provided evidence that the hippo pathway, especially its core effector YAP, played a key role in upregulating nasal epithelial proliferation and remodelling in nasal polyps, which suggested the hippo pathway could be a valuable therapeutic target in nasal polyps. However, how the hippo pathway regulates the pro-inflammatory effect of epithelial cells is still unknown. Thus, to uncover key insights of the control of hyperinflammatory responses elicited from epithelial cells in CRSwNP, we identified the possible pathway in epithelial proliferation and epithelium-derived innate cytokines production. Here, we found that YAP overexpression induced the upregulation of the epithelium-derived innate cytokines TSLP, IL-33, and IL-25 via NF- $\mathrm{B}$ signalling, consistent with other studies that NF- $\kappa B$ played an important role in $\mathrm{CRS}^{12}$ These novel findings provide new insights into the pathophysiology of inflammation in nasal polyps.

In this study, we also showed that aberrant expression of YAP induced epithelial cell proliferation during nasal polyp development. It was related to cell cycle, because when the hippo pathway was downregulated, the cells were arrested in G1 phase resulting in repressed cell growth. Consistent with our findings, previous study found that YAP-deficient retinal progenitors displayed decreased S-phase cells and altered cell cycle progression. ${ }^{17}$ Furthermore, another study demonstrated that when YAP was decreased in cells from clear cell renal cell carcinoma, cell proliferation was inhibited (G1 phase arrest) and apoptosis was increased. ${ }^{18}$

As reported, nasal epithelial cells played a key role in producing a series of pro-inflammatory cytokines such as TSLP, IL-25, and IL-33 that orchestrated type 2 responses at the mucosa site. ${ }^{19,20}$ IL-33 and TSLP mRNA levels were increased in polyp tissues. ${ }^{21}$ In this study, we revealed that nasal polyp tissue had increased mRNA levels of the epithelium-derived innate cytokines TSLP, IL-25, and IL-33. However, the mechanism by which cytokine expression is upregulated has not been well documented. Several studies have demonstrated that 
A

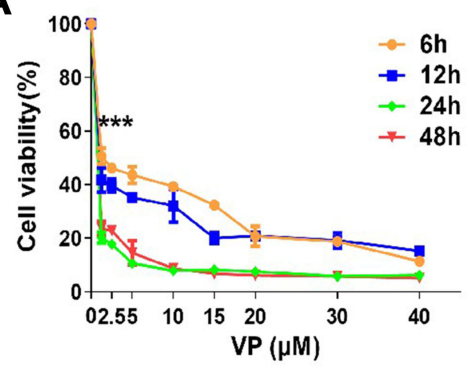

D

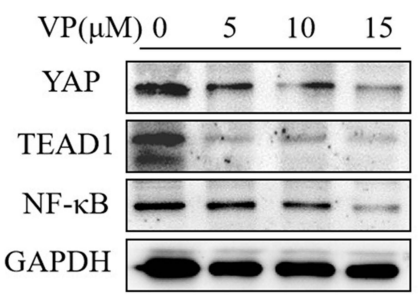

H
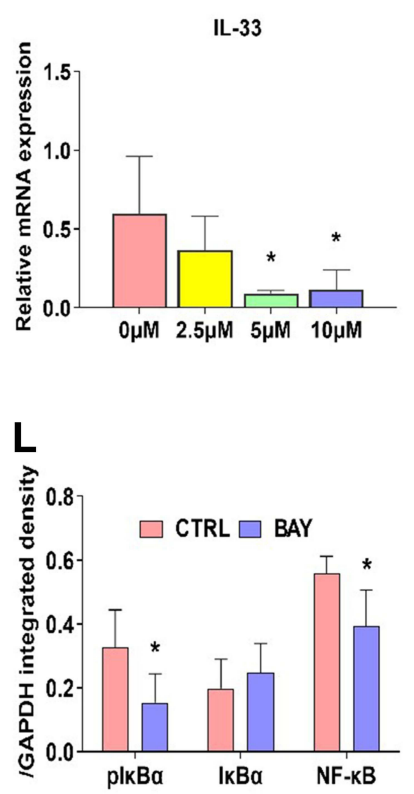

E

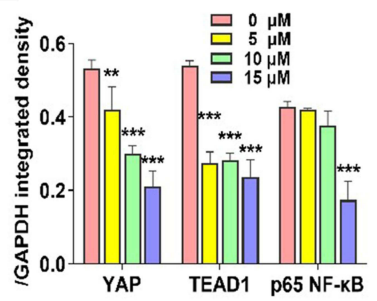

I

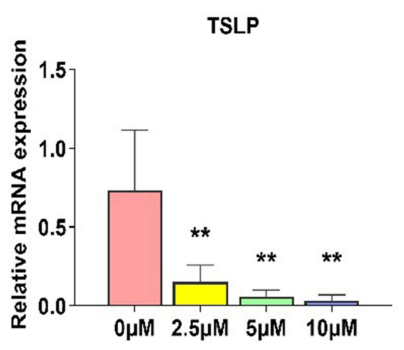

M

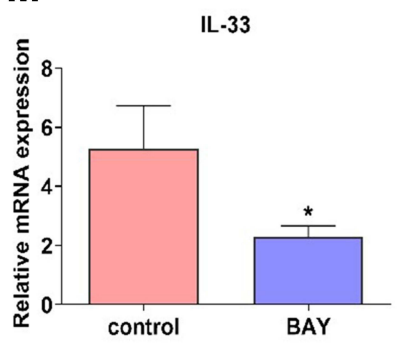

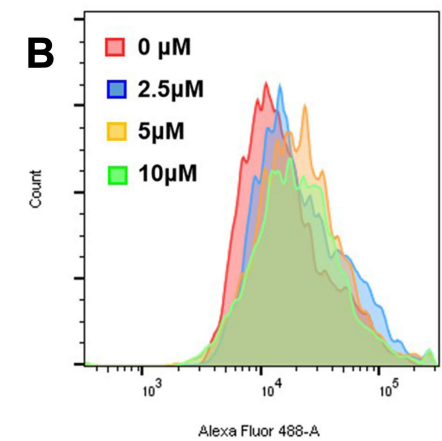

F

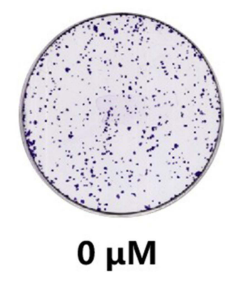

J

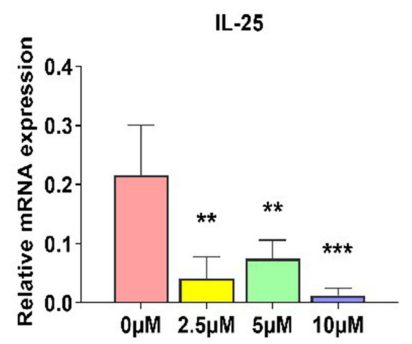

$\mathbf{N}$

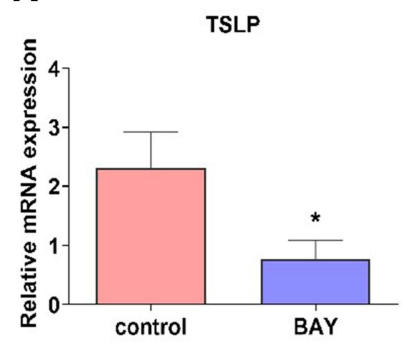

C

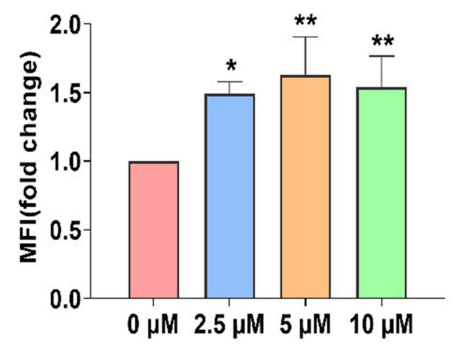

G

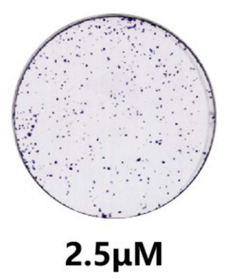

K

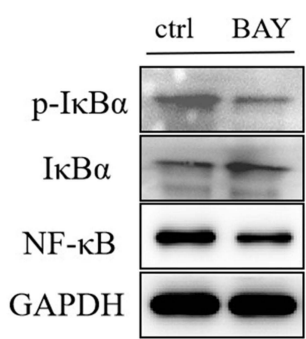

0

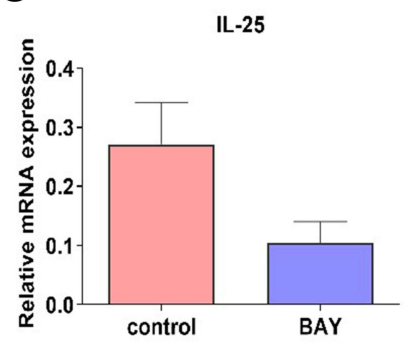

Figure 6 Suppression of cell proliferation by inhibition of YAP was mediated by NF- $\mathrm{kB}$. (A) Cell viability of NPECs treated with verteporfin from $6 \mathrm{~h}$ to $48 \mathrm{~h}$ in different density gradient $(0 \mu \mathrm{M}-40 \mu \mathrm{M})$, respectively $(n=3)$. (B-C) Flow cytometry of cell proliferation with VP treatment in cells from human nasal polyps. The cells were cultured with VP for $24 \mathrm{~h}$ after CFSE staining. Quantification of MF in various conditions was shown ( $\mathrm{n}=3$ for each group, one-way ANOVA). (D-E) Quantification of YAP, TEADI and 665 NF- $\kappa B$ expression after stimulation with VP for $24 \mathrm{~h}$ by Image $\mathrm{J}$ software ( $\mathrm{n}=3$ for each group, one-way ANOVA). (F-G) The biological behavior changes of the NPECs were observed by colony formation assay. Representative images from each group are shown ( $\mathrm{n}=3$ for each group, Independent-Sample $t$ Test). (H-J) Quantitative analysis of mRNA levels of TSLP, IL-33 and IL-25 from primary nasal polyp epithelial cells treated with VP for $24 \mathrm{~h}$ ( $\mathrm{n}=3$, Kruskal-Wallis H-test). (K-L) NPECs was incubated with BAY $(5 \mu \mathrm{M})$ for $24 \mathrm{~h}$. Western blotting was performed to measure the expression of the key protein levels of NF- $\mathrm{kB}$ pathway $(\mathrm{n}=3)$. $(\mathbf{M}-\mathrm{O})$ The $\mathrm{mRNA}$ expression levels of TSLP, IL-33 and IL-25 were detected relative to the expression level of $\beta 2 M$ in NPECs incubated with or without BAY $(5 \mu M)$ for $24 \mathrm{~h}(\mathrm{n}=3$, Mann-Whitney U 2-test). Results are the mean $\pm \mathrm{SD}$. $* P<0.05$, $* * P<0.01$, $* * * P<0.001$.

the NF- $\kappa$ B pathway is highly activated in inflammatory disorders due to its ability to induce transcription of proinflammatory genes. ${ }^{22,23}$ Similar to another study, ${ }^{10}$ we found that $\mathrm{NF}-\mathrm{KB}$ activity involved in the production of epithelium-derived innate cytokines TSLP and IL-33, as well as IL-25 expression in epithelia cells from nasal polyps. Possibly, when an inflammatory process is initiated in nasal polyps, activated NF-kB translocates 
to the cell nucleus, inducing transcription, particularly of genes encoding inflammatory cytokines, chemokines, and adhesion molecules, such as TSLP, IL-33, and IL25. This $N F-\kappa B$-driven effect was further verified in a pharmacological inhibition experiment that nasal polyp epithelial cells treated with BAY 11-7082 (5 $\mu \mathrm{M})$ could reduce TSLP, IL-33, and IL-25 expression.

YAP functions as a transcriptional regulator by acting together with sequence-specific DNA binding factors and transcription cofactors to mediate cell proliferation in developing epithelial tissues and tumours. Meanwhile, it has been reported that NF- $\mathrm{B}$ and NF- $\mathrm{BB}$-related inflammatory cytokines are increased in nasal polyps, indicating that NF- $\kappa B$ has a pivotal role in the pathogenesis of CRSwNPs in Asians. ${ }^{10}$ In the present study, we found that inhibition of YAP could reduce the NF- $\kappa$ B pathway. The interaction between YAP and NF- $\mathrm{B}$ was supported by many studies. One study underscored the importance of the YAP1/TAZ-NFאB signalling axis in endodermal organ development and disease, and they found that Hippo suppressed NF- $\mathrm{BB}$ signalling in pancreatic progenitors to permit cell differentiation and epithelial morphogenesis. ${ }^{24}$ Besides, others found that YAP enhanced NF- $\kappa$ B-dependent and independent effects on clock-mediated unfolded protein responses and autophagy in sarcoma. ${ }^{25}$ Similarly, in our study, YAP-induced upregulation of TSLP, IL-33, and IL-25 expression was abrogated by NF- $\kappa \mathrm{B}$ inhibition, indicating that YAP could induce epithelial cytokine production in nasal epithelial cells via activation of the NF- $\kappa$ B pathway. Importantly, VP could be a potential treatment for inhibition of inflammation in nasal polyps by downregulating NF- $\kappa \mathrm{B}$ signalling pathway.

\section{Conclusions}

Our study confirmed that inhibition of YAP could downregulate the expression of TSLP, IL-33, and IL-25 via the $\mathrm{NF}-\kappa \mathrm{B}$ signalling pathway in nasal epithelial cells. It provided the possible evidence that clinically YAP inhibition could serve as a potential therapeutic approach for treating nasal inflammation, such as using verteporfin (a selective YAP inhibitor verteporfin) to reduce the mucosal inflammation and recurrence. However, further studies are warranted to characterize the exact mechanism of YAP and NF- $\mathrm{BB}$ in the regulation of inflammatory cell functions in nasal polyps.

\section{Ethics Approval and Informed Consent}

All patients were informed of the purposes and procedures of the study and provided written informed consent. This study was approved by the Ethics Committee for Human Study at the Third Affiliated Hospital of Sun Yat-sen University (China).

\section{Consent for Publication}

We confirm that the details of any images, videos, recordings, etc can be published, and that the persons providing consent have been shown the article contents to be published.

\section{Acknowledgments}

The authors would like to thank all the patients for their participation in this study, as well as the funding, and The Third Affiliated Hospital, Sun Yat-sen University.

\section{Author Contributions}

All authors made substantial contributions to conception and design, acquisition of data, or analysis and interpretation of data; took part in drafting the article or revising it critically for important intellectual content; agreed to submit to the current journal; gave final approval of the version to be published; and agree to be accountable for all aspects of the work.

\section{Funding}

This work was supported by the National Natural Science Foundation of China (Grant No. 82000957 and 81870704), the Science and Technology Planning Project of Guangdong Province (2014A020212057) and The Third Affiliated Hospital of Sun Yat-Sen University, Clinical Research Program (No. QHJH201901).

\section{Disclosure}

The authors declare that the research was conducted in the absence of any commercial or financial relationships that could be construed as a potential conflict of interest.

\section{References}

1. Shi JB, Fu QL, Zhang H, et al. Epidemiology of chronic rhinosinusitis: results from a cross-sectional survey in seven Chinese cities. Allergy. 2015;70(5):533-539. doi:10.1111/all.12577

2. Watelet JB, Van Zele T, Gjomarkaj M, et al. Tissue remodelling in upper airways: where is the link with lower airway remodelling? Allergy. 2006;61(11):1249-1258. doi:10.1111/j.1398-9995.2006.01226.x

3. Van Bruaene N, Van Crombruggen K, Van Crombruggen K, et al. Inflammation and remodelling patterns in early stage chronic rhinosinusitis. Clin Exp Allergy. 2012;42(6):883-890. doi:10.1111/ j.1365-2222.2011.03898.x

4. Brescia G, Alessandrini L, Marioni G. Structured histopathology for endotyping and planning rational treatment in chronic rhinosinusitis. $\mathrm{Am}$ J Otolaryngol. 2021;42(1):102795. doi:10.1016/j.amjoto.2020.102795 
5. Johnson RL. Hippo signaling and epithelial cell plasticity in mammalian liver development, homeostasis, injury and disease. Sci China Life Sci. 2019;62(12):1609-1616. doi:10.1007/s11427-018-9510-3

6. Mitani A, Nagase T, Fukuchi K, Aburatani H, Makita R, Kurihara H. Transcriptional coactivator with PDZ-binding motif is essential for normal alveolarization in mice. Am J Respir Crit Care Med. 2009;180(4):326-338. doi:10.1164/rccm.200812-1827OC

7. Lange AW, Sridharan A, Xu Y, Stripp BR, Perl AK, Whitsett JA. Hippo/Yap signaling controls epithelial progenitor cell proliferation and differentiation in the embryonic and adult lung. $\mathrm{J} \mathrm{Mol} \mathrm{Cell} \mathrm{Biol.}$ 2015;7(1):35-47. doi:10.1093/jmcb/mju046

8. Deng $\mathrm{H}$, Sun $\mathrm{Y}$, Wang $\mathrm{W}$, et al. The hippo pathway effector Yes-associated protein promotes epithelial proliferation and remodeling in chronic rhinosinusitis with nasal polyps. Allergy. 2019;74 (4):731-742. doi:10.1111/all.13647

9. Chen X, Chang L, Li X, et al. Tc17/IL-17A Up-Regulated the Expression of MMP-9 via NF-kappaB Pathway in Nasal Epithelial Cells of Patients With Chronic Rhinosinusitis. Front Immunol. 2018;9:2121. doi:10.3389/fimmu.2018.02121

10. Jung HJ, Zhang YL, Kim DK, Rhee CS, Kim DY. The Role of NF-kappaB in Chronic Rhinosinusitis With Nasal Polyps. Allergy Asthma Immunol Res. 2019;11(6):806-817. doi:10.4168/aair.2019. 11.6 .806

11. Xu R, Xu G, Shi J, Wen W. A correlative study of NF-kappaB activity and cytokines expression in human chronic nasal sinusitis. J Laryngol Otol. 2007;121(7):644-649. doi:10.1017/S00222151 06001824

12. Kim YH. Establishing a Therapeutic Strategy Targeting NF-kappaB in Asian Patients with Chronic Rhinosinusitis With Nasal Polyps. Allergy Asthma Immunol Res. 2019;11(6):757-759. doi:10.4168/ aair.2019.11.6.757

13. Fokkens WJ, Lund VJ, Mullol J, et al. 2012: European position paper on rhinosinusitis and nasal polyps 2012. A summary for otorhinolaryngologists. Rhinology. 2012;50(1):1-12. doi:10.4193/ Rhino50E2

14. Rosenbluh J, Nijhawan D, Cox AG, et al. beta-Catenin-driven cancers require a YAP1 transcriptional complex for survival and tumorigenesis. Cell. 2012;151(7):1457-1473. doi:10.1016/j.cell.20 12.11 .026

15. Zhang GC, Yu XN, Sun JL, et al. UBE2M promotes cell proliferation via the beta-catenin/cyclin D1 signaling in hepatocellular carcinoma. Aging. 2020;12(3):2373-2392. doi:10.18632/aging.102 749
16. Mjosberg JM, Trifari S, Crellin NK, et al. Human IL-25- and IL-33responsive type 2 innate lymphoid cells are defined by expression of CRTH2 and CD161. Nat Immunol. 2011;12(11):1055-1062. doi:10. 1038/ni.2104

17. Kim JY, Park R, Lee JH, et al. Yap is essential for retinal progenitor cell cycle progression and RPE cell fate acquisition in the developing mouse eye. Dev Biol. 2016;419(2):336-347. doi:10.1016/j. ydbio.2016.09.001

18. Cao JJ, Zhao XM, Wang DL, et al. YAP is overexpressed in clear cell renal cell carcinoma and its knockdown reduces cell proliferation and induces cell cycle arrest and apoptosis. Oncol Rep. 2014;32 (4):1594-1600. doi:10.3892/or.2014.3349

19. Lloyd CM, Saglani S. Epithelial cytokines and pulmonary allergic inflammation. Curr Opin Immunol. 2015;34:52-58. doi:10.1016/j. coi.2015.02.001

20. Zheng R, Wang D, Wang K, et al. Elevated expression of IL-17RB and ST2 on myeloid dendritic cells is associated with a Th2-skewed eosinophilic inflammation in nasal polyps. Clin Transl Allergy. 2018;8:50. doi:10.1186/s13601-018-0237-4

21. Hong HY, Chen FH, Sun YQ, et al. Local IL-25 contributes to Th2-biased inflammatory profiles in nasal polyps. Allergy. 2018;73 (2):459-469. doi:10.1111/all.13267

22. Tak PP, Firestein GS. NF-kappaB: a key role in inflammatory diseases. J Clin Invest. 2001;107(1):7-11. doi:10.1172/JCI11830

23. Yamamoto Y, Gaynor RB. Therapeutic potential of inhibition of the NF-kappaB pathway in the treatment of inflammation and cancer. J Clin Invest. 2001;107(2):135-142. doi:10.1172/JCI11914

24. Braitsch CM, Azizoglu DB, Htike Y, et al. LATS1/2 suppress NFkappaB and aberrant EMT initiation to permit pancreatic progenitor differentiation. Plos Biol. 2019;17(7):e3000382. doi:10.1371/journal. pbio. 3000382

25. Rivera-Reyes A, Ye S. YAP1 enhances NF-kappaB-dependent and independent effects on clock-mediated unfolded protein responses and autophagy in sarcoma. Cell Death Dis. 2018;9(11):1108. doi:10.1038/s41419-018-1142-4

\section{Publish your work in this journal}

The Journal of Asthma and Allergy is an international, peer-reviewed open-access journal publishing original research, reports, editorials and commentaries on the following topics: Asthma; Pulmonary physiology; Asthma related clinical health; Clinical immunology and the immunological basis of disease; Pharmacological interventions and new therapies. The manuscript management system is completely online and includes a very quick and fair peer-review system, which is all easy to use. Visit http://www.dovepress.com/testimonials.php to read real quotes from published authors. 